\title{
Disfunção Autonômica Cardiovascular no Diabetes Mellitus Experimental
}

\begin{abstract}
RESUMO
Muitas informações novas têm sido publicadas nos últimos anos a respeito da fisiopatologia da disfunção autonômica cardiovascular em ratos e camundongos diabéticos. Nosso grupo tem estudado o curso temporal das alterações cardiovasculares associadas ao diabetes experimental há alguns anos, obtendo evidências consistentes de grave disautonomia em modelos animais de diabetes. O objetivo deste trabalho foi revisar a contribuição que estudos envolvendo diferentes modelos de deficiência e resistência à insulina têm fornecido para o entendimento, tratamento e prevenção da disfunção autonômica cardiovascular do diabetes. (Arq Bras Endocrinol Metab 2007;51/2:185-194)
\end{abstract}

Descritores: Diabetes mellitus; Neuropatia autonômica; Disfunção cardiovascular; Modelos animais

\begin{abstract}
Cardiovascular Autonomic Dysfunction in Experimental Diabetes Mellitus.

Much new information has been published in the last few years regarding pathophysiology of cardiovascular autonomic dysfunction in diabetic rats and mice. Our group has been studying the time-course cardiovascular changes associated with experimental diabetes in the last years, and obtained consistent evidences of severe dysautonomia in diabetes animal models. The aim of this manuscript is to review the contribution that studies involving different animal models of insulin deficiency or resistance have given to understand, treat and prevent diabetic cardiovascular autonomic dysfunction. (Arq Bras Endocrinol Metab 2007;51/2:185-194)
\end{abstract}

Keywords: Diabetes mellitus; Autonomic neuropathy; Cardiovascular dysfunction; Animal models

Pow OR VOLTA DE 1920, Walter Cannon, o patriarca das ciências fisiológicas, dividiu o sistema nervoso autônomo em simpático e parassimpático. Ele propôs que os dois sistemas se antagonizavam para manter a "homeostase funcional", termo criado pelo próprio autor (1). O conceito fisiopatológico da disfunção autonômica é relativamente novo na medicina clínica, possivelmente datado dos anos 20, quando Bradburry e Eggleston estudaram as causas neurogênicas da hipotensão postural (2). Em termos gerais, a disautonomia refere-se à condição em que, na presença da função autonômica alterada, esta afetará negativamente a saúde do indivíduo, oscilando desde pequenos episódios transitórios até a uma progressiva doença neurodegenerativa (3).

A neuropatia autonômica (NA) é uma importante complicação do diabetes. No entanto, apesar de sua relação com o aumento do risco para

\section{revisão}

\author{
Katia De Angells \\ BEATRIZ D'AGORD SCHAAN \\ BRUNO RODRIGUES \\ Christiane Malfitano \\ MARIA CLÁUdIA IRIGOYEN
}

Universidade São Judas Tadeu

(KDA) e Unidade de

Hipertensão, Instituto

do Coração (InCor), Faculdade

de Medicina, Universidade

de São Paulo (BR, CM \& MCl),

São Paulo, SP; e Serviço

de Medicina Experimental

da Unidade de Pesquisa do

Instituto de Cardiologia,

Fundação Universitária de

Cardiologia, Porto Alegre, RS. 
mortalidade cardiovascular (4) e sua associação com múltiplos sintomas e prejuízos funcionais (5), ainda não existem dados totalmente conclusivos sobre vários de seus aspectos. É de amplo conhecimento que as complicações crônicas microvasculares do diabetes ocorrem tardiamente e estão associadas a anormalidades estruturais de nervos, rins, retina e vasos, além de se associarem ao tempo de doença e mau controle glicêmico $(6,7)$. A prevalência da NA, no entanto, varia amplamente na dependência da população estudada e dos métodos de investigação empregados. Em indivíduos assintomáticos, observou-se que até $20 \%$ deles apresentaram prejuízo da função autonômica cardiovascular. Esta informação foi enfatizada em recente publicação pela American Diabetes Association, que destaca a importância da neuropatia autonômica no curso temporal da doença (8).

A NA cardiovascular é a forma mais estudada e clinicamente importante dentre as neuropatias diabéticas. Estudos demonstraram que a disfunção autonômica cardiovascular, medida através da variabilidade da freqüência cardíaca, está associada a um aumento do risco (duas vezes maior) de isquemia miocárdica silenciosa e mortalidade $(9,10)$. Estas complicações são comumente encontradas tanto no diabetes tipo 1 quanto no tipo 2.

Nosso grupo tem estudado as alterações cardiovasculares associadas ao diabetes experimental de forma temporal há alguns anos. O objetivo deste trabalho foi revisar a contribuição dos estudos experimentais nas alterações funcionais do sistema cardiovascular de diferentes modelos animais de diabetes.

\section{MODELOS DE DEFICIÊNCIA DE INSULINA}

O diabetes experimental induzido por estreptozotocina (STZ) tem sido amplamente utilizado no estudo da relação entre o diabetes e desordens autonômicas cardiovasculares. A STZ destrói as células $\beta$ pancreáticas resultando em uma síndrome com características similares àquelas encontradas em seres humanos com diabetes tipo l, isto é, hiperglicemia, hipoinsulinemia, glicosúria, poliúria, polidipsia e perda de peso (11-17). Em relação aos parâmetros cardiovasculares, ratos tratados com STZ apresentam redução ou manutenção da pressão arterial (PA) quando comparados a animais controles, alterações que são observadas desde 5 dias após a injeção da droga $(12,13,16-18)$. O fato do diabetes descompensado ser um estado de depleção de volume pode contribuir para a redução da PA destes animais. De fato, a inibição da NO-sintase pelo uso de L-NAME (19) e a sobrecarga salina crônica (20) determinaram, neste modelo, aumentos maiores de PA do que nos animais não-diabéticos, possivelmente por mecanismos volume-dependente e de sensibilidade exagerada ao sal. Além disto, bradicardia de repouso tem sido observada nos animais tratados com STZ, a qual tem sido atribuída a alterações no nodo sinusal $(12,17,18,21)$.

Considerando outras espécies, como por exemplo camundongos, um dos modelos animais mais utilizados para induzir modificações genéticas, são poucas as informações disponíveis, embora estejam documentadas mudanças na PA e prejuízos na função vascular e renal após o tratamento com STZ (22-24). Além disso, autores sugerem que os efeitos da STZ em camundongos sobre a PA possam estar relacionados a fatores genéticos $(23,24)$. Resultados de nosso laboratório com camundongos C57BL/SV129 híbridos mostraram que, diferentemente do que se observa em ratos, o diabetes por $\mathrm{STZ}$ se associa à hipertensão e à hiperglicemia, sem alterações na FC (24). Por outro lado, a hipertensão do diabetes por STZ estava ausente em camundongos knockout (KO) para os receptores ATl de Angiotensina II (ANG II) (24), sugerindo que o sistema renina-angiotensina (SRA) seja determinante no aumento da $\mathrm{PA}$, à semelhança do observado previamente em estudos clínicos.

Devemos enfatizar que a $\mathrm{PA}$ e a $\mathrm{FC}$ basais não necessariamente fornecem informações diretas a respeito da função autonômica. Para o acompanhamento das interações entre as diferentes alças do sistema nervoso autônomo no diabetes, faz-se necessária a quantificação de marcadores da função autonômica pelo uso de ferramentas que permitam avaliar a variabilidade da FC e da PA, as respostas ao bloqueio autonômico induzidas por fármacos, as alterações funcionais no baro e quimiorreflexo, além de alterações da estrutura dos órgãos acometidos.

Em ratos tratados com STZ encontramos redução do tônus vagal, acompanhada de manutenção do tônus simpático para o coração $(12,17,18)$, demonstrada por redução do tônus vagal central, uma vez que a estimulação direta dos nervos eferentes produziu bradicardia até maior nos diabéticos do que a observada nos controles. A disfunção parassimpática observada no diabetes por STZ pode estar relacionada a alterações nos receptores muscarínicos cardíacos (25), o que pode ser testado pela resposta à estimulação colinérgica. Para tal, a metacolina tem sido utilizada como um agonista muscarínico. A resposta bradicárdica induzida pela metacolina mostrou-se aumentada após a indução do diabetes por STZ 
$(13,26)$, sugerindo que uma hipersensibilidade dos receptores pudesse estar ligada à redução da atividade parassimpática observada nestes animais. De fato, recentemente demonstramos que o diabetes por STZ está associado a um aumento da função muscarínica no átrio (27). A disfunção parassimpática foi também estudada em ratos diabéticos induzidos por aloxano, um modelo químico de diabetes que se assemelha a STZ e que provoca deficiência de insulina (28).

O controle barorreflexo da circulação é um dos mais importantes mecanismos de controle momento a momento da pressão arterial. De fato, o controle da variabilidade da pressão arterial pelo barorreflexo é a chave para a homeostase fisiológica. Isso é demonstrado clinicamente em estudos que mostraram que o prejuízo da função barorreflexa é um fator de risco independente para morte súbita pós-infarto do miocárdio (29). Além disso, pacientes diabéticos com reflexos cardiovasculares normais têm menor mortalidade quando comparados àqueles que apresentam algum tipo de prejuízo na função reflexa autonômica (4). Estudos têm demonstrado que a atividade dos barorreceptores pode modular tanto a atividade simpática como a parassimpática para vasos e coração, indicando que alterações nas vias aferentes e/ou eferentes do barorreflexo podem estar diferencialmente envolvidas na disfunção autonômica do diabetes.

Muitos estudos usando modelos experimentais de diabetes têm investigado os mecanismos de disfunção no controle neural (11-18,25,30,31). Estudos de nosso grupo mostraram que pouco tempo após a indução do diabetes por STZ, os animais já apresentavam prejuízo no controle reflexo da PA, especialmente para a resposta taquicárdica às quedas da $\mathrm{PA}$ $(16,31)$. Cronicamente, a bradicardia reflexa também se mostrou alterada, comprometendo assim as duas alças barorreflexas $(11,12,32)$. A disfunção barorreflexa tem sido atribuída a alterações no parassimpático cardíaco $(13,17)$, embora alterações na função do receptor, ou mesmo na mediação central do reflexo, não possam ser excluídas.

Adicionalmente, existem evidências de que o diabetes gestacional também afeta a função barorreflexa da prole. Em filhotes de mães diabéticas por STZ, além da disfunção barorreflexa, observou-se um aumento da PA e da atividade tecidual da enzima conversora de angiotensina (ECA) (33).

Outro importante sub-conjunto de aferências é aquele representado pelos quimiorreceptores arteriais, os quais também participam no controle autonômico cardiovascular, porém têm sido muito menos estudados. O quimiorreflexo, testado através da infusão de cianeto de potássio (KCN), o qual estimula as vias simpáticas (levando à hipertensão) e parassimpáticas (induzindo bradicardia), está atenuado em ratos diabéticos por STZ (13). O reconhecimento de prejuízo no controle desses reflexos na condição diabética abre espaço para intervenções que possam ser usadas no manejo da disfunção do quimio e do barorreflexo, entre as quais está o treinamento físico, ferramenta não farmacológica que tem sido utilizada com múltiplos benefícios cardiovasculares. Em nosso laboratório, demonstramos que 10 semanas de treinamento físico em esteira rolante reverteu as alterações hemodinâmicas e recuperou a sensibilidade do baro e do quimiorreflexo observadas em ratos diabéticos por STZ $(18,32)$.

Outro grupo importante de aferências que contribui para o controle autonômico cardiovascular e que se encontra prejudicado em ratos diabéticos por STZ é o dos receptores cardiopulmonares. Esses receptores são importantes na regulação do balanço de fluidos, via modulação simpática sistêmica e renal $(33,34)$. Em animais de experimentação, esse reflexo é estimulado por injeção de serotonina, promovendo bradicardia e hipotensão. Não foram encontradas modificações em resposta a serotonina em animais diabéticos por STZ (33), sugerindo que o reflexo cardiopulmonar estivesse preservado neste modelo experimental. Entretanto, a resposta reflexa induzida pela expansão de volume plasmático associada a alterações na pressão diastólica final, que normalmente se associa à redução da atividade simpática renal, está abolida em animais diabéticos quando comparados com os controles (33). O papel fisiológico dessa resposta alterada no diabetes pode representar um ajuste frente à disfunção no balanço de sódio e água, bem como alterações nas respostas diuréticas e natriuréticas desta condição. A redução da excreção renal de sódio associada à diminuição da ativação simpática já foi descrita previamente (34).

Outro indicador da modulação autonômica do sistema cardiovascular, a variabilidade da FC e da PA estimadas no domínio do tempo ou da freqüência (análise espectral), tem sido usado para detectar anormalidades da função autonômica do sistema cardiovascular em diferentes circunstâncias (35-37), com a vantagem de ser uma ferramenta não-invasiva e de fácil aplicação em humanos. Mudanças nessas variáveis podem ser detectadas mesmo que não se observem alterações da PA e da FC basais. Por exemplo, a redução da variabilidade da FC está associada a um aumento do risco de morte súbita em pacientes com insuficiência cardíaca crônica (38). Além do mais, em 
indivíduos diabéticos há uma significante diminuição da variabilidade da FC, avaliada por análise espectral, mesmo na ausência de outras manifestações nos parâmetros cardiovasculares (36). A importância da variabilidade da PA em patologias clínicas foi estabelecida através de estudos que demonstravam que o seu aumento estava associado a lesões de órgãos-alvo (39).

Os mecanismos de mudanças na variabilidade da FC em pacientes diabéticos podem estar relacionados à neuropatia autonômica ou lesão nos nervos parassimpáticos. Dessa forma, o estudo da variabilidade da FC é uma poderosa ferramenta para verificar a integridade funcional do sistema parassimpático cardíaco. Fazan e cols. demonstraram que uma redução da variabilidade da FC estava associada à diminuição dos componentes de baixa freqüência (LF), indicador de modulação simpática, e alta freqüência (HF), indicador de modulação parassimpática. Em estudo com ratos diabéticos por STZ, os autores demonstraram que o desvio-padrão dos pulsos pressóricos adjacentes (um índice da variabilidade da FC) estava reduzido nesses animais quando comparados aos controles (30). Estes achados foram confirmados através da variação da variabilidade da FC e da PA em ratos diabéticos por STZ (15). Essas alterações estão correlacionadas negativamente com os níveis de glicose plasmática, sugerindo que o aumento dos níveis glicêmicos esteja associado ao prejuízo da função autonômica do coração nesse modelo (15). Reforçando esta hipótese, o tratamento com insulina em ratos com diabetes por STZ melhorou o perfil hemodinâmico desses animais (normalização da PA e FC), além de aumentar a variabilidade da FC. De acordo com esses estudos, e em acordo com estudos clíni$\cos (6)$, a disfunção autonômica de pacientes com diabetes pode ser revertida com um bom controle metabólico $(40,41)$.

Em camundongos, entretanto, os resultados foram diferentes: o diabetes por STZ não se associou com alterações na FC e na sua variabilidade $(23,24)$. Porém, houve uma diminuição da variabilidade da PA, com alteração do componente LF, o que se acredita esteja relacionado a um aumento da modulação simpática $(37,42)$. Interessantemente, a redução da variabilidade da PA induzida pelo diabetes não ocorre em animais $\mathrm{KO}$ para os receptores de angiotensina II (ATl) (24). Isso indica, mais uma vez, o papel dos receptores de angiotensina II na modulação autonômica observada no diabetes (43). Estes dados confirmam achados prévios em animais diabéticos por STZ que também mostraram alterações da variabilidade da PA $(15,35)$.
Outro modelo animal de deficiência de insulina é o rato $\mathrm{BB} / \mathrm{W}$ (Bio-Bred), no qual a deficiência ocorre espontaneamente durante seu amadurecimento. Os sintomas têm início abrupto, incluindo perda de peso, hipoinsulinemia, hiperglicemia e cetonúria (44). Ratos diabéticos $\mathrm{BB} / \mathrm{W}$ são normotensos, porém apresentam alteração da sensibilidade do barorreflexo. Não se observou diferenças na resposta reflexa bradicárdica à infusão de fenilefrina, porém a resposta reflexa taquicárdica ao nitroprussiato de sódio foi mais evidente nestes animais (45), sugerindo uma potencialização dessa resposta. Entretanto, não existem dados sobre o controle autonômico cardiovascular ou sobre os reflexos comandados pelos receptores cardiopulmonares ou pelos quimiorreceptores. Sinais de neuropatia autonômica diabética foram também detectados nos nervos intramurais de átrios e ventrículos dos animais espontaneamente diabéticos (BB) (46). Estas alterações histopatológicas podem ser responsáveis por mudanças na via barorreflexa, prejudicando a condução do impulso nervoso. Esses achados são consistentes com resultados obtidos em animais diabéticos por STZ que mostraram origem neurogênica para a redução da resposta cardiovascular reflexa $(13,16,32)$.

Outro modelo interessante é o dos animais espontaneamente diabéticos (WBN/Kob), que também têm sido estudados por desenvolver hiperglicemia, glicosúria, poliúria, hipoinsulinemia e intolerância à glicose a partir dos 9 meses de idade até o 17 mês em machos, pois as fêmeas não apresentam esse mesmo perfil de alterações (47). Avaliações histopatológicas revelaram alterações no pâncreas de ratos machos a partir de 3 meses de idade. Com o avanço da idade, o tecido fibroso gradualmente invade muitas áreas do pâncreas, inclusive as ilhotas, que acabam envolvidas nessa degeneração fibrótica (48).

Ratos WBN/Kob entre 4 a 5 e 8 a 9 meses de idade apresentam diminuição significante da FC, sem alteração de PA quando comparados com ratos Wistar da mesma idade. Esses animais com 4 a 5 meses apresentam um desbalanço simpato-vagal em favor do simpático, pela análise da relação LF/HF, provavelmente originada pelo aumento da modulação simpática. Nesse período, os animais mostraram diminuição dos níveis plasmáticos de insulina, porém sem alteração dos níveis de glicose. Os achados acima sugerem que os animais encontram-se em um estado pré-diabético observado também pelo desbalanço simpato-vagal. Os animais WNB/Kob de 8 a 9 meses apresentam hiperglicemia com redução nas concentrações plasmáticas de insulina, ausência do ritmo circadiano da FC e aumento da PA durante o sono, como previamente 
demonstrado em seres humanos (49), além de disfunção autonômica, caracterizada por aumento da modulação simpática, que provavelmente precede uma redução da atividade parassimpática em animais $\mathrm{WBN} / \mathrm{Kob}(50)$. As respostas reflexas cardiovasculares mediadas pelo sistema nervoso autônomo ainda não foram estudadas neste modelo.

Outro modelo animal de deficiência de insulina que surgiu mais recentemente é o dos camundongos diabéticos não-obesos (NOD), originalmente isolados por uma mutação espontânea. Os animais desenvolvem o diabetes através de uma reação auto-imune das células T nas ilhotas pancreáticas, um processo que se origina aproximadamente entre 4 a 8 semanas de idade, com maior incidência em fêmeas $(80 \%)$ do que em machos (20\%) (51). Os camundongos NOD são hipoinsulinêmicos, hiperglicêmicos e necessitam de administração de insulina para sobreviver. Esses animais têm pouco tempo de vida, de 5 a 8 semanas após o início do diabetes. Esses camundongos apresentam complicações características do diabetes $(52,53)$, desenvolvendo rapidamente neuropatia no gânglio simpático paravertebral (53). É importante salientar que existe um prejuízo maior no sistema nervoso autônomo em camundongos NOD quando comparados a ratos ou camundongos diabéticos por STZ (53), sugerindo uma importante disfunção autonômica cardiovascular. Entretanto, informações sobre disfunção dos reflexos autonômicos neste modelo ainda não estão disponíveis na literatura.

\section{MODELOS CARACTERIZADOS POR RESISTÊNCIA À INSULINA E INTOLERÂNCIA À GLICOSE}

Os mecanismos relacionados ao diabetes tipo 2 e sua associação com patologias cardiovasculares têm sido elucidados através do uso de modelos experimentais de obesidade e diabetes, tais como os ratos obesos Zucker e os camundongos db/db e ob/ob. O objetivo é ter modelos animais que mais se aproximam da condição humana, na qual a obesidade é fator muito comum associado ao diabetes tipo 2 .

No modelo de Zucker, descrito primeiramente em 1960 (54), uma mutação no gene do receptor da leptina resulta em prejuízo na capacidade da enzima em suprimir a ingestão de alimento. Estão presentes hiperinsulinemia, dislipidemia, hiperfagia, hipotermia, alterações na atividade simpática e redução do gasto energético (54-57). Há, porém, resultados contraditórios quanto às características cardiovasculares e autonômicas nesse modelo. Alguns estudos mostram hipertensão e atividade simpática aumentada no rato Zucker obeso, quando comparado ao magro $(56,57)$. Entretanto, outros estudos mostram pressão arterial normal $(58,59)$ e redução da atividade simpática no mesmo modelo (60). Embora a função cardiovascular venha sendo muito investigada no modelo, ainda existem poucos estudos que incluem avaliação da glicemia, tolerância à glicose ou insulina (57,61-63). Portanto, é difícil concluir diretamente a relação entre o metabolismo de glicose e a função cardiovascular nesse modelo. Ruggeri e cols. encontraram aumento no reflexo somato-simpático em ratos Zucker obesos resistentes à insulina. Nesse estudo, os animais eram normotensos, sugerindo que a reatividade simpática aumentada e a resistência à insulina estavam presentes antes do início da hipertensão (64). Esses resultados contraditórios levaram alguns autores a buscar evidências das alterações na PA. A medida direta da PA, realizada em animais conscientes, demonstrou que eles não eram de fato hipertensos. Entretanto, sob anestesia, a PA estava aumentada e o bloqueio ganglionar autonômico normalizava essa diferença, sugerindo que o tônus simpático aumentado poderia ser responsável pela elevação da PA, embora deva-se considerar que o uso de anestesia tenha dificultado a interpretação desses dados (58).

Com o objetivo de monitorar a PA com maior precisão em ratos conscientes, Carlson e cols. usaram a radio-telemetria para comparar ratos Zucker diabéticos e obesos a ratos Zucker não-diabéticos magros ( 12 semanas de idade). Os autores demonstraram que os animais diabéticos obesos tinham hipertensão leve (aumento de aproximadamente $13 \mathrm{mmHg}$ na PA). $\mathrm{O}$ bloqueio ganglionar com hexametônio causou uma grande diminuição na PA dos animais obesos, sugerindo a participação de um exacerbado tônus simpático na elevação da PA neste modelo (57).

A função vasodilatadora (induzida por acetilcolina) também foi menor nos ratos Zucker obesos $(58,65)$. É importante enfatizar que em ratos Zucker obesos normotensos observou-se prejuízo da resposta barorreflexa cronotrópica, sugerindo que a disfunção barorreflexa possa estar contribuindo para o estabelecimento da hipertensão nesse modelo (66).

A modulação autonômica cardíaca em ratos Zucker obesos e magros ( 10 semanas de idade), avaliada pela análise espectral dos sinais do monitoramento eletrocardiográfico de 24 horas, demonstrou que a FC foi menor nos animais obesos quando comparados aos animais magros. No entanto, as bandas de HF, de LF e o balanço LF/HF foram similares entre 
os ratos Zucker obesos e magros, sendo que o ritmo circadiano desses parâmetros, na maior parte, estava preservado. Além disso, o efeito do bloqueio autonômico farmacológico (atropina e propranolol) sobre a FC foi semelhante nos ratos obesos e magros. Esses resultados sugerem que a variabilidade da FC, avaliada pela análise espectral, é normal em ratos Zucker obesos resistentes à insulina (67). No entanto, não existem dados sobre o quimiorreflexo, o reflexo cardiopulmonar ou sobre a variabilidade de PA nesse modelo.

Com a introdução na dieta do xarope de milho rico em frutose, principal açúcar utilizado para adoçar alimentos industrializados, no final de 1960, o consumo de frutose na dieta americana aumentou notavelmente (68), o que contribui para o desenvolvimento de resistência à insulina, obesidade, hipertensão e anormalidades lipídicas (69). A partir desses dados, a busca por modelos animais com sobrecarga de frutose e que levassem a alterações semelhantes passou a motivar os pesquisadores da área. De fato, ratos alimentados com frutose mostraram moderada hipertensão e intolerância à glicose, associados a níveis plasmáticos aumentados de insulina, colesterol e triglicérides (70-72). Em camundongos, o consumo crônico de frutose alterou o metabolismo de glicose e o perfil lipídico $(37,73)$.

Nosso grupo, utilizando a radio-telemetria, demonstrou recentemente que oito semanas de dieta rica em frutose foram suficientes para induzir intolerância à glicose associada à hipertensão noturna (período ativo dessa espécie) em camundongos. Houve também um aumento da variabilidade da PA sistólica (três vezes) e das oscilações da banda de LF no espectro da variabilidade da PA (duas vezes) durante o período escuro. No entanto, a dieta de frutose não mudou a variabilidade do intervalo de pulso, analisada no domínio do tempo e da freqüência, nem a sensibilidade barorreflexa (37). Estes resultados não só corroboram dados clínicos que demonstraram, em pacientes diabéticos hipertensos, aumento na variabilidade da PA (74), como também podem ser relevantes para o entendimento das alterações fisiopatológicas iniciais do diabetes tipo 2 em humanos. Os mecanismos subjacentes às mudanças cardiovasculares induzidas pelo consumo de frutose ressaltam o papel do sistema nervoso simpático e do sistema renina-angiotensina (SRA). A simpatectomia (medulectomia adrenal associada à exposição com neurotoxina) atenuou o desenvolvimento da hipertensão nos ratos alimentados com dieta rica em frutose (75). Além disso, o consumo de frutose aumentou a excreção urinária de catecolaminas e a expressão de receptores adrenérgicos (71). Esses achados em conjunto reforçam o papel do sistema nervoso simpático na gênese da hipertensão. As evidências do papel do SRA nas mudanças cardiovasculares induzidas pela sobrecarga de frutose foram observadas pela expressão aumentada dos receptores de angiotensina II nos vasos, pelo efeito depressor dos antagonistas dos receptores ATl $(70,72)$ e pela ativação do SRA vascular (73). Demonstramos, também, um aumento dos níveis plasmáticos de angiotensina II e mudanças no sistema nervoso central, observadas quando aumenta a expressão do RNAm dos receptores ATla cerebrais (37). Recentemente, estudando a participação do receptor ATla da angiotensina II nas alterações cardiovasculares e metabólicas induzidas pelo consumo de frutose, evidenciamos que a PA não estava aumentada em camundongos knockout para o receptor ATla, além de esses animais apresentarem melhor perfil metabólico quando comparados a camundongos controles submetidos ao mesmo tempo de dieta rica em frutose. Esse resultado nos permitiu sugerir que, mesmo em presença de ativação do SRA, a ausência do receptor ATla provavelmente desvia a utilização da ANG II para vias vasodilatadoras, o que justificaria a redução da PA observada nos animais knockout para esses receptores. Esses achados confirmam aqueles obtidos com o uso dos antagonistas dos receptores ATl da ANG II em indivíduos diabéticos e estão de acordo com a idéia de que o bloqueio do receptor ATla altera a tolerância à glicose (37).

A ativação do SRA observada em ratos alimentados com frutose poderia ser um dos mecanismos de elevação da PA por sua ação vasoconstrictora direta ou pelo aumento da reabsorção de sódio. Entretanto, existem evidências de que a elevação da PA está associada à hiperinsulinemia mais do que à retenção de sódio ou ao aumento no volume plasmático em ratos tratados com frutose (76). Além do mais, nem a atividade simpática renal, nem a resposta reflexa à expansão de volume estavam alterados nesses animais (77).

Outros modelos com diferentes tipos de dieta também têm sido usados para avaliar a relação entre obesidade e/ou resistência insulínica e a disfunção cardiovascular. O desenvolvimento da hipertensão leve em ratos obesos decorrente da ingestão de dieta rica em gordura foi detectado primeiramente pela medida indireta da pressão na cauda e confirmado posteriormente pela medida direta intra-arterial. Embora a taquicardia reflexa às quedas da PA não diferisse entre os grupos, a bradicardia reflexa em resposta aos aumentos de PA estava atenuada nos obesos em relação aos controles, e isso foi atribuído à deficiente mediação parassimpática (65). 
Camundongos C57BL/6J (BL/6), da mesma linhagem da qual foram originados os camundongos obesos ob/ob, se tornam diabéticos, hiperinsulinêmicos e obesos quando alimentados a partir do desmame com uma dieta rica em gordura e carboidratos simples (bigh fat-high simple carbohydrate - HFHSC), sendo esse mais um modelo de estudo do diabetes tipo 2 (78). Entretanto, seus controles, os camundongos A/J, alimentados com a mesma dieta, tornam-se obesos e levemente resistentes à insulina, mas não diabéticos. Mills e cols. evidenciaram que a dieta HFHSC $+0,06 \%$ de sódio induziu aumento dos níveis plasmáticos de insulina em camundongos $\mathrm{A} / \mathrm{J}$ e $\mathrm{BL} / 6$, mas somente camundongos $\mathrm{BL} / 6$ desenvolveram hipertensão (79). Aparentemente na interação entre DM tipo 2 e hipertensão nos animais $\mathrm{BL} / 6$, estão envolvidas alterações genéticas que podem aumentar a atividade simpática associada à alimentação rica em carboidratos e gorduras (80). De fato, o bloqueio ganglionar nesses animais sugere um excessivo recrutamento simpático para vasos e rins que deve contribuir para o desenvolvimento de hipertensão e das alterações metabólicas em camundongos BL/6 alimentados com dieta HFHSC (78). Não foram encontrados dados referentes ao controle do reflexo cardiovascular, variabilidade da PA e da FC em animais submetidos a dieta rica em gordura e carboidratos.

Nos camundongos geneticamente diabéticos $\mathrm{db} / \mathrm{db}$, os resultados da mutação da leptina levam à hiperglicemia - em níveis semelhantes ao modelo de diabetes-STZ - à resistência à insulina e hiperinsulinemia (81). Esse animal tem sido usado como um modelo de diabetes tipo 2 , porém são poucos os estudos que avaliaram a disfunção cardiovascular e autonômica nesse modelo. A pressão arterial avaliada de forma indireta de 11 a 27 semanas de idade mostrou-se inalterada $(82,83)$, mas a PA sistólica e média estavam aumentadas em camundongos $\mathrm{db} / \mathrm{db}$ com $\sim 12$ semanas de idade, associada a aumento da resistência periférica total e FC normal (84). Há estudos que mostram a presença da neuropatia periférica nesse modelo, iniciada poucos meses após o nascimento. A concentração de norepinefrina (NE) mostrou-se reduzida no coração, rins e glândulas salivares dos camundongos $\mathrm{db} / \mathrm{db}$ (24 semanas de idade) (85). Os átrios de camundongos $\mathrm{db} / \mathrm{db}$ foram hipersensíveis à noradrenalina exógena, no entanto responderam à estimulação nervosa colinérgica e ACh exógena de uma maneira similar aos átrios de animais não-diabéticos. As respostas dos vasos à estimulação nervosa em animais $\mathrm{db} / \mathrm{db}$ foram similares à de animais normais (86). Esses achados indicam algum grau de disfunção autonômica nesse modelo.
Camundongos geneticamente obesos $K K A^{y}$ e $o b / o b$ são modelos que recentemente começaram a ser estudados sob o aspecto cardiovascular. Os camundongos $K K A^{y}$ apresentam níveis aumentados de leptina plasmática e pressão arterial sistólica aumentada medida de forma indireta, além de elevada excreção urinária de catecolaminas e exacerbada resposta a antagonistas simpáticos em comparação a camundongos controles, sugerindo uma relação papel do simpático na hipertensão associada a esse modelo de obesidade (87). No entanto, medidas diretas da pressão arterial em camundongos $\mathrm{ob} / \mathrm{ob}$ em registros diretos de curta duração $(88)$ ou contínuos por radiotelemetria (89) demonstraram que esses animais não apresentavam níveis elevados de pressão arterial. Todavia, não existem estudos detalhados sobre o controle autonômico da circulação nesses modelos geneticamente modificados.

\section{CONSIDERAÇŌES FINAIS}

São muitas as evidências de que o sistema nervoso autônomo desempenha um papel-chave na fisiopatologia das complicações do diabetes mellitus, tanto em humanos como em modelos animais. Entre as diferentes formas de neuropatia, a disfunção autonômica cardiovascular, associada com mau prognóstico, incluindo aumento da morbi-mortalidade cardiovascular, tem sido uma das entidades clínicas muito estudadas. O estudo do comportamento da pressão arterial e da freqüência cardíaca, bem como do controle autonômico da circulação em modelos animais de diabetes, tem contribuído para o entendimento dos mecanismos fisiopatológicos envolvidos durante $\mathrm{o}$ curso temporal da disfunção metabólica. Além disso, novas abordagens, como a utilização de animais geneticamente modificados, poderão fornecer informações inovadoras a respeito da participação de diferentes mecanismos e/ou sistemas, como o sistema renina-angiotensina no delineamento dos fatores ambientais e genéticos envolvidos no desenvolvimento da disautonomia do diabetes. $\mathrm{O}$ conhecimento dessas associações poderá encorajar médicos, profissionais da saúde e também pacientes na busca de intervenções, farmacológicas ou comportamentais, que precocemente melhorem não só o controle metabólico, mas também a disfunção autonômica.

\section{REFERÊNCIAS}

1. Cannon WB. The wisdom of the body. New York: W.W. Norton, 1939.

2. Bradbury S, Eggleston C. Postural hypotension. A report of three cases. Am Heart J 1925;1:73-86. 
3. Goldstein DS, Robertson D, Esler M, Straus SE, Eisenhofer G. Dysautonomias: clinical disorders of the autonomic nervous system. Ann Intern Med 2002;137(9):753-63.

4. Ewing D, Campbell I, Clarke B. The natural history of diabetic autonomic neuropathy. Quart J Med 1980;49:95-108.

5. Vinik Al, Maser RE, Mitchell BD, Freeman R. Diabetic autonomic neuropathy. Diabetes Care 2003;26(5):1553-79.

6. The Diabetes Control and Complications Trial Research Group. The effect of intensive treatment of diabetes on the development and progression of long-term complications in insulin-dependent diabetes mellitus. N Eng J Med 1993;329:977-86.

7. UK Prospective Diabetes Study (UKPDS) Group. Intensive blood-glucose control with sulphonylureas or insulin compared with conventional treatment and risk of complications in patients with type 2 diabetes (UKPDS 33). Lancet 1998;352:837-53.

8. Boulton AJM, Vinik Al, Arezzo JC, Bril V, Feldman EL, Freeman R, et al. Diabetic neuropathies: a statement by the American Diabetes Association. Diabetes Care 2005;28:956-62.

9. Ewing DJ, Martyn CN, Young RJ, Clarke BF. The value of cardiovascular autonomic function tests: 10 years experience in diabetes. Diabetes Care 1985;8:491-8.

10. Sawicki PT, Kiwitt S, Bender R, Berger M. The value of QT interval dispersion for identification of total mortality risk in non-insulin-dependent diabetes mellitus. J Intern Med 1998;243:49-56.

11. Tomlinson KC, Sheila MG, Anhony-Hebden R, Bennett $T$. Functional consequences of streptozotocin-induced diabetes mellitus, with particular reference to the cardiovascular system. Pharmacol Rev 1981;44:103-48.

12. De Angelis K, Schaan BD, Maeda CY, Dall'Ago P, Wichi RB, Irigoyen MC. Cardiovascular control in experimental diabetes. Braz J Med Biol Res 2002;35(9):1091-100.

13. Dall'Ago P, Fernandes TG, Machado UF, Belló AA, Irigoyen MC. Baroreflex and chemoreflex dysfunction in streptozotocin-diabetic rats. Braz J Med Biol Res 1997;30:119-24.

14. Schaan BD, Maeda CY, Timm H, Medeiros S, Moraes R, Ferlin $E$, et al. Time course of changes in heart rate and blood pressure variability in streptozotocin-induced diabetic rats treated with insulin. Braz J Med Biol Res 1997;30:1081-6.

15. Schaan BD, Dall'Ago $P$, Maeda CY, Ferlin E, Fernandes TG, Schmid $\mathrm{H}$, et al. Relationship between cardiovascular dysfunction and hyperglycemia in streptozotocin-induced diabetes in rats. Braz J Med Biol Res 2004;37:1895-902.

16. Maeda CY, Fernandes TG, Lulhier F, Irigoyen MC. Streptozotocin diabetes modifies arterial pressure and baroreflex sensitivity in rats. Braz J Med Biol Res 1995;28:497-501.

17. Maeda CY, Fernandes TG, Timm HB, Irigoyen MC. Autonomic dysfunction in short-term experimental diabetes. Hypertension 1995;26(Part 2):1000-4.

18. De Angelis KLD, Oliveira AR, Dall'Ago $P$, Peixoto LRA, Gadonski G, Fernandes TG, et al. Effects of exercise training in autonomic and myocardial dysfunction in streptozotocin-diabetic rats. Braz J Med Biol Res 2000;33:635-41.

19. Balbinott AW, Irigoyen MC, Brasileiro-Santos S, Zottis B, De Lima NG, Passaglia J, et al. Dose-dependent autonomic dysfunction in chronic L-NAME-hypertensive diabetic rats. J Card Pharm 2005;46(5):563-9.

20. Maeda CY, Schaan BD, Oliveira VL, De Angelis K, Oliveira EM, Irigoyen MC. Chronic salt loading and cardiovascular associated changes in experimental diabetes. Clin Exp Pharmacol Physiol 2006;in press.

21. Senges J, Brachmann J, Pelzer D, Hasslacher C, Weihe E, Kübler W. Altered cardiac automaticity and conduction in experimental diabetes mellitus. J Mol Cell Card 1980;12:1341-51.

22. Katoh M, Ohmachi Y, Kurosawa Y, Yoneda H, Tanaka N, Narita $\mathrm{H}$. Effects of imidapril and captopril on streptozotocininduced diabetic nephropathy in mice. Eur J Pharmacol 2000;398(3):381-7.

23. Gurley SB, Clare SE, Snow KP, Hu A, Meyer TW, Coffman TM. Impact of genetic background on nephropathy in diabetic mice. Am J Physiol Renal Physiol 2006;290(1):214-22.
24. Wichi RB, Farah V, Chen Y, Irigoyen MC, Morris M. Deficiency in angiotensin AT1a receptors prevents diabetes-induced hypertension. Am J Physiol Regul Integr Comp Physiol 2006; in press.

25. Carrier GO, Aronstam RS. Altered muscarinic receptor properties and function in the heart in diabetes. J Pharm Exper Therap 1987;242:531-5.

26. Li X, Tanz RD, Chang KSK. Effect of age and methacholine on the rate and coronary flow of isolated hearts of diabetic rats. Br J Pharm 1989;97:1209-17.

27. Dall'Ago P, D'Agord Schaan B, da Silva VO, Werner J, da Silva Soares PP, De Angelis K, et al. Parasympathetic dysfunction is associated with baroreflex and chemoreflex impairment in streptozotocin-induced diabetes in rats. Auton Neurosci 2007;30:28-35.

28. Kuntscherova J, VIk J. Influence of alloxan diabetes on acetylcholine synthesis in tissues of the albino rat. Physiology Bohemoslov 1970;19(5):431-4.

29. La Rovere MT, Bigger JT Jr, Marcus FI, Mortara A, Schwartz PJ. Baroreflex sensitivity and heart-rate variability in prediction of total cardiac mortality after myocardial infarction. ATRAMI (Autonomic Tone and Reflexes After Myocardial Infarction) Investigators. Lancet 1998;351(9101):478-84.

30. Fazan R, Ballejo G, Salgado MC, Moraes M, Salgado HC. Heart rate variability and baroreceptor function in chronic diabetic rats. Hypertension 1997;30(Part 2):632-5.

31. Page $M$, Watkins P. Cardiorespiratory arrest and diabetic autonomic neuropathy. Lancet 1978;7(1):14-6.

32. Harthmann AD, De Angelis K, Parente-Costa L, Senador $D$, Schaan BD, Krieger EM, et al. Exercise training improves arterial baro and chemoreflex in control and diabetic rats. Auton Neur B Clin 2007; in press.

33. Oliveira VLL, Moreira ED, Farah V, Consolim-Colombo F, Krieger EM, Irigoyen MC. Cardiopulmonary reflex impairment in experimental diabetes in rats. Hypertension 1999;34:813-7.

34. Wichi RB, Souza SB, Casarini DE, Morris M, Barreto-Chaves $\mathrm{ML}$, Irigoyen MC. Increased blood pressure in the offspring of diabetic mothers. Am J Physiol Regul Integr Comp Physiol 2005;288:R1129-33.

35. Patel KP, Zhang PL. Reduced renal sympathoinhibition in response to acute volume expansion in diabetic rats. Am J Phys 1994;267:R372-9.

36. Fazan R Jr, Dias da Silva VJ, Ballejo G, Salgado HC. Power spectra of arterial pressure and heart rate in streptozotocininduced diabetes in rats. J Hypertens 1999;17:489-95.

37. Pagani M, Malfatto G, Pierini S, Casati R, Masu AM, Poli M, et al. Spectral analysis of heart rate variability in the assessment of autonomic diabetic neuropathy. J Auton Nerv Syst 1988; $23: 143-53$

38. Farah V, Elased KM, Chen Y, Key MP, Cunha TS, Irigoyen MC, et al. Nocturnal hypertension in mice consuming a high fructose diet. Auton Neurosci 2006;130(1-2):41-50.

39. Galinier M, Pathak A, Fourcade J, Androdias C, Curnier D, Varnous S, et al. Depressed low frequency power of heart rate variability as an independent predictor of sudden death in chronic heart failure. Eur Heart J 2000;21(6):475-82.

40. Zanchetti A, Mancia G. Blood pressure and organ damage. J Cardiovasc Pharmacol 1987;10(6):S111-8.

41. Hreidarsson AB. Acute, reversible autonomic nervous system abnormalities in juvenile insulin-dependent diabetes: A pupillographic study. Diabetologia 1981;20:475-81.

42. Ferreira SR, Cesarini PR, Vivolo MA, Zanella MT. Abnormal nocturnal blood pressure fall in normotensive adolescents with insulin-dependent diabetes is ameliorated following glycemic improvement. Braz J Med Biol Res 1998;31:5238.

43. Stauss HM, Mrowka R, Nafz B, Patzak A, Unger T, Persson PB. Does low frequency power of arterial blood pressure reflect sympathetic tone? J Auton Nerv Syst 1995;54(2):145-54.

44. Chen $Y$, Joaquim LF, Farah VM, Wichi RB, Fazan R Jr., Salgado $\mathrm{HC}$, et al. Cardiovascular autonomic control in mice lacking angiotensin AT1a receptors. Am J Physiol Regul Integr Comp Physiol 2005;288:R1071-77. 
45. Crisa L, Mordes JP, Rossini AA. The etiology is related to an autoimmune dysfunction. Diabetes Metab Rev 1992;8:9-37.

46. Krizsan-Agbas D, Buñag RD. Normotensive diabetic BB/W rats show enhanced reflex tachycardia. Diabetes 1991;40:1504-10.

47. Addicks K, Boy C, Rosen P. Sympathetic autonomic neuropathy in the heart of the spontaneous diabetic BB rat. Ann Anat 1993;175(3):253-7.

48. Hashimoto M, Harada T, Ishikawa T, Obata M, Shibutani $Y$. Investigation on diabetic autonomic neuropathy assessed by power spectral analysis of heart rate variability in WBN/Kob rats. J Electrocardiol 2001;34(3):243-50.

49. Nakama K, Shichinohe K, Kobayashi K, Naito K, Uchida O, Yasuhara K, et al. Spontaneous diabetes-like syndrome in WBN/Kob rats. Acta Diabet Lat 1985;22:335-42.

50. Madacsy L, Yasar A, Tulassay T, Korner A, Kelemen J, Hobor $M$, et al. Relative nocturnal hypertension in children with insulin-dependent diabetes mellitus. Acta Paediatr 1994;83(4):414-7.

51. Van Buren T, Schiereck P, De Ruiter GJ, Gispen WH, De Wildt DJ. Vagal efferent control of electrical properties of the heart in experimental diabetes. Acta Diabetol 1998;35(1):19-25.

52. Kikutani $\mathrm{H}$, Makino $\mathrm{S}$. The murine autoimmune diabetes model: NOD and related strains. Adv Immunol 1992;51:285-322.

53. Watkins CC, Sawa A, Jaffrey S, Blackshaw S, Barrow RK, Sny$\operatorname{der} \mathrm{SH}$, et al. Insulin restores neuronal nitric oxide synthase expression and function that is lost in diabetic gastropathy. $\mathbf{J}$ Clin Invest 2000;106:373-84.

54. Schmidt RE, Dorsey LA, Beaudet LN, Frederick KE, Parvin CA, Plurad SB, et al. Non-obese diabetic mice rapidly develop dramatic sympathetic neuritic dystrophy a new experimental model of diabetic autonomic neuropathy. Am J Pathology 2003;163(5):2077-91.

55. Zucker LM, Zucker TF. Hereditary obesity in the rat associated with high serum fat and cholesterol. Proc Sot Exp Biol Med 1962;110:165-71.

56. Bray GA, York DA. Studies on food intake of genetically obese rats. Am J Physiol 1972;223:176-9.

57. Turner NC, Gudgeon C, Toseland N. Effects of genetic hyperinsulinaemia on vascular reactivity, blood pressure, and renal structure in the Zucker rat. J Cardiovasc Pharmacol 1995;26:714-20.

58. Carlson SH, Shelton J, White CR, Wyss JM. Elevated sympathetic activity contributes to hypertension and salt sensitivity in diabetic obese Zucker rats. Hypertension 2000;35:403-8.

59. Pamidimukkala J, Jandhyala BS. Evaluation of hemodynamics, vascular reactivity and baroreceptor compensation in the insulin resistant Zucker obese rats. Clin Exp Hypertens 1996;18:1089-104.

60. Pawloski CM, Kanagy NL, Mortensen LH, Fink GD. Obese Zucker rats are normotensive on normal and increased sodium intake. Hypertension 1992;19:190-5.

61. Levin BE, Triscari J, Sullivan AC. Studies of origins of abnormal sympathetic function in obese Zucker rats. Am J Physiol Endocrinol Metab 1983;245:E87-93.

62. Buchanan TA, Sipos GF, Madrilejo N, Chaplin L, Campese VM. Hypertension without peripheral insulin resistance in spontaneously hypertensive rats. Am J Physiol Endocrinol Metab 1992;262:E14-9.

63. De Angelis KLD, Gadonski G, Fang J, Dall'Ago P, Albuquerque VL, Peixoto LRA, et al. Exercise reverses peripheral insulin resistance in trained L-NAME-hypertensive rats. Hypertension 1999;34(2):768-72.

64. Ferrannini E, Buzzigoli G, Bonadonna R, Giorico MA, Oleggini M, Graziadei L, et al. Insulin resistance in essential hypertension. N Engl J Med 1987;317:350-7.

65. Ruggeri P, Brunori A, Cogo CA, Storace D, Di Nardo F, Burattini $R$. Enhanced sympathetic reactivity associates with insulin resistance in the young Zucker rat. Am J Physiol Regul Integr Comp Physiol 2006;291(2):R376-82.

66. Bunag RD, Eriksson L, Krizsan D. Baroreceptor reflex impairment and mild hypertension in rats with dietary-induced obesity. Hypertension 1990;15(4):397-406.
67. Bunag RD, Barringer DL. Obese Zucker rats, though still normotensive, already have impaired chronotropic baroreflexes. Clin Exp Hypertens [A] 1988;10(1):257-62.

68. Towa S, Kuwahara M, Tsubone H. Characteristics of autonomic nervous function in Zucker-fatty rats: investigation by power spectral analysis of heart rate variability. Exp Anim 2004;53(2):137-44.

69. Putnam J, Allshouse J, Kantor LS. U.S. per capita food supply trends: more calories, refined carbohydrates and fats. Food Review 2002;25:2-15.

70. Basciano H, Federico L, Adeli K. Fructose, insulin resistance, and metabolic dyslipidemia. Nutr Metab 2005;2(1):5.

71. Katovich MJ, Reaves PY, Francis SC, Pachori AS, Wang HW, Raizada MK. Gene therapy attenuates the elevated blood pressure and glucose intolerance in an insulin-resistant model of hypertension. J Hypertens 2001;19:1553-8.

72. Kamide K, Rakugi H, Higaki J, Okamura A, Nagai M, Moriguchi $\mathrm{K}$, et al. The renin-angiotensin and adrenergic nervous system in cardiac hypertrophy in fructose-fed rats. Am J Hypertens 2002;15:66-71.

73. Hsieh PS. Reversal of fructose-induced hypertension and insulin resistance by chronic losartan treatment is independent of AT2 receptor activation in rats. $J$ Hypertens 2005;23:2209-17.

74. Nagata R, Nishio Y, Sekine O, Nagai Y, Maeno Y, Ugi S, et al. Single nucleotide polymorphism (-468 Gly to $A)$ at the promoter region of SREBP-1c associates with genetic defect of fructose-induced hepatic lipogenesis [corrected]. J Biol Chem 2004;279:29031-42.

75. Shinozaki K, Ayajiki K, Nishio Y, Sugaya T, Kashiwagi A, Okamura $T$. Evidence for a causal role of the renin-angiotensin system in vascular dysfunction associated with insulin resistance. Hypertension 2004;43:255-62.

76. Mancia G, Ferrari A, Gregorini L, Parati G, Pomidossi G, Bertinieri $G$, et al. Blood pressure and heart rate variabilities in normotensive and hypertensive human beings. Circ Res 1983;53:96-104.

77. Verma S, Bhanot S, McNeill JH. Sympathectomy prevents fructose-induced hyperinsulinemia and hypertension. Eur J Pharmacol 1999;373:R1-4.

78. Lyev SN, Katovich MJ. Fructose feeding in rats is not associated with sodium retention. Am J Hypert 1996;9:1018-23.

79. Wong Patrick SK, Johns EJ. Effect of acute saline volume loading on renal sympathetic nerve activity in anaesthetised fructose-fed and fat-fed rats. J Auton Nerv Syst 1999;75:60-9.

80. Surwit RS, Kuhn CM, Cochrane D, McCubbin JA, Feinglos MS. Diet induced type II diabetes in C57BL/6J mice. Diabetes 1988;37:1163-6

81. Mills $E$, Kuhn CM, Feinglos MN, Surwit R. Hypertension in CB57BL/6J mouse model of non-insulin-dependent diabetes mellitus. Am J Physiol 1993;264(1 Pt 2):R73-8.

82. Landsberg $L$, Young JB. Fasting, feeding and the regulation of the sympathetic nervous system. N Engl J Med 1978;298:1295-301.

83. Chen $\mathrm{H}$, Charlat O, Tartaglia LA, Woolf EA, Weng $X$, Ellis SJ, et al. Evidence that the diabetes gene encodes the leptin receptor: identification of a mutation in the leptin receptor gene in db/db mice. Cell 1996;84:491-5.

84. Koya D, Haneda M, Nakagawa H, Isshiki K, Sato H, Maeda S, et al. Amelioration of accelerated diabetic mesangial expansion by treatment with a PKC beta inhibitor in diabetic $\mathrm{db} / \mathrm{db}$ mice, a rodent model for type 2 diabetes. FASEB J 2000;14(3):439-47.

85. Moriyama T, Oka K, Ueda H, Imai E. Nilvadipine attenuates mesangial expansion and glomerular hypertrophy in diabetic $\mathrm{db} / \mathrm{db}$ mice, a model for type 2 diabetes. Clin Exp Nephrol 2004;8(3):230-6.

86. Bagi Z, Erdei N, Toth A, Li W, Hintze TH, Koller A, et al. Type 2 diabetic mice have increased arteriolar tone and blood pressure: enhanced release of COX-2-derived constrictor prostaglandins. Arterioscler Thromb Vasc Biol 2005; 25(8):1610-6. 
87. Giachetti A. The functional state of sympathetic nerves in spontaneously diabetic mice. Diabetes 1978;27(10):969-74.

88. Wilson HF, Mayer JH, Clarke SA, Tomlinson DR. An examination of autonomic nervous function in genetically diabetic mice. J Auton Pharmacol 1982;2(3):147-53.

89. Aizawa-Abe M, Ogawa Y, Masuzaki H, Ebihara K, Satoh N, Iwai $\mathrm{H}$, et al. Pathophysiological role of leptin in obesity-related hypertension. J Clin Invest 2000;105:1243-52.

90. Mark AL, Shaffer R, Correia M, Morgan D, Sigmund $C$ Haynes WG. Contrasting blood pressure effects of obesity in leptin-deficient ob/ob mice and agouti yellow obese mice. J Hypertens 1999;17:1949-53.
91. Swoap SJ. Altered leptin signaling is sufficient, but not required, for hypotension associated with caloric restriction. Am J Physiol Heart Circ Physiol 2001;281(6):H2473-9.

Endereço para correspondência:

Maria Cláudia Irigoyen

Unidade de Hipertensão - Incor, HC-FMUSP

Av. Enéas Carvalho de Aguiar 44

05403-000 São Paulo, SP

Fax: (1 1 ) 3085-7887

E-mail: hipirigoyen@incor.usp.br 